\title{
Effect of dielectrophoretic force in the self-assembly process of electrosprayed nanoparticles ${ }^{1}$
}

\author{
L.Castañer ${ }^{2}$, S.Bermejo and M. Dominguez-Pumar \\ UPC, modul C4 Campus Nord UPC, Jordi Girona 1, Barcelona 08034, Spain
}

\begin{abstract}
In this work the effect of the dielectrophoretic force (DEP) in the selfassembly process of nanoparticles electrosprayed onto a substrate, is examined. DEP force is originated by the electric field created by the electrospray gun and by the distortion of the field created by the effective dipole of each nanoparticle. It is also shown that the modulus of this force is large when the distance between particles is few times its diameter, provided the medium is wet and the electric field is not switched off.

The directional nature of DEP In this wet phase, is shown to chain nanoparticles aligned with the main electric field direction. Although there is a repulsive force between chains in the orthogonal direction to the field, it is minimum when the beads align with the voids in the nearby chains.

DEP is a dominant force in the close distances of nanoparticles compared to double layer, van der Waals, electrophoretic retardation, weight and buoyancy.
\end{abstract}

Keywords: Nanoparticles, Electrospray, Self-assembly

\section{Introduction}

Nanoparticles are used in a number of applications ranging from the smart textiles, as antibacterial agent [1] to a mean to enhance the capacitance of a supercapacitor [2] or to create heat management schemes useful for thermal sensors and thermocrystals [3]. Not only passive functionality is searched but also a more active role in the operation of electronic or photonic devices

\footnotetext{
${ }^{1}$ Work funded by MINECO project ESP2014-54256-C4-2-R

${ }^{2}$ corresponding author luis.castaner@upc.edu
} 


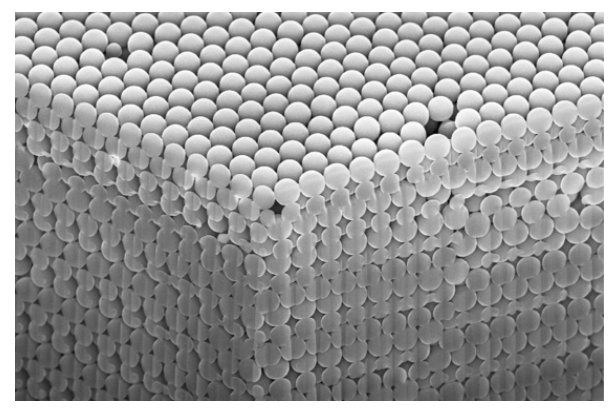

Figure 1: Close view of an electrospray deposit of latex nanospheres

[4]. One of the barriers hindering the mass production of devices based on nanoparticles, is the deposition technology itself, relying most of the times in capillary forces: Langmuir-Blodgett [5], wedge cell [6], spin-on [7] or dip coating [8]. We have recently shown [9] the potential of electrospray to deposit nanoparticles in large areas and short times. Electrospray is a cheap and easily scalable technique having a high material deposition efficiency and directivity [10]. Although electrospray is a well established technique, broadly used in many applications such as ion mass spectroscopy [13], it has not been used to deposit nanoparticles out of a colloidal solution until recently [14] [9]. In our previous works, we deposited by electrospray layers of both polystyrene and $\mathrm{SiO}_{2}$ nanoparticles. To our surprise, when a set of experimental conditions were met, the layer of deposited nanoparticles showed an hexagonal close packing arrangement (hcp) and, in large areas, face centered cubic (fcc). An example can be seen in Figure (1) where a SEM picture shows not only the surface but also the organization in depth.

This result demonstrated that the electrospray technique has a great potential in depositing thick and large area layers of self-assembled nanoparticles compared to other methods that either, take a much longer time, or produce only monolayers.

We noticed, in our experiments, that such an ordered self-assembly was only possible if the high applied voltage was kept ON until evaporation of the solvent was totally completed. Moreover, we observed that the aerosol droplets should leave some liquid on the surface as, when the deposit is too dry, no significant order in the layer is observed. Finally we observed that the pumping rate and the conductivity of the liquid had a minor impact. Details are given in references [9],[15] and [16]. 


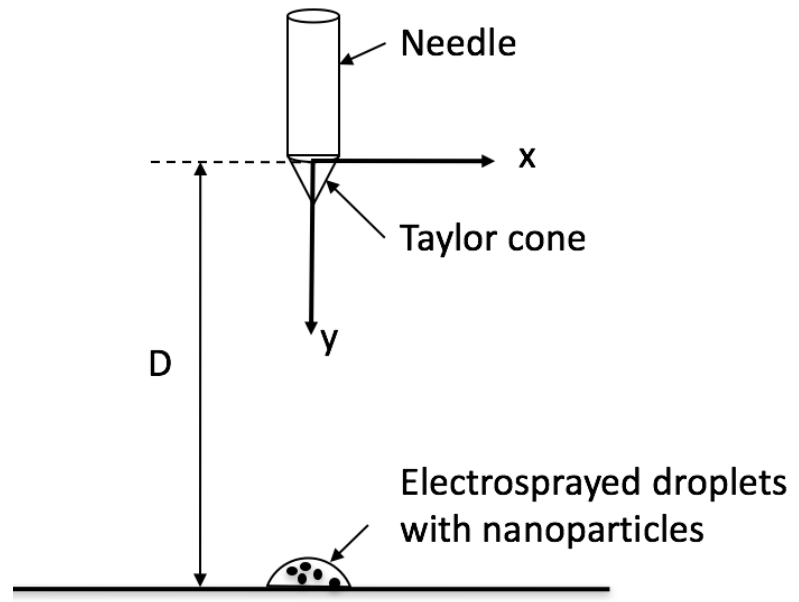

Figure 2: Schematic description of the electrospray geometry used in this works

In this paper, the importance of the dielectrophoretic force in the self assembly process is described. In Section 2 an analytical model of the DEP force is developed for a simple geometry and attention os paid to the modulus of the force and interestingly to the direction. The results are qualitatively validated with numerical simulations. As the DEP force is the main difference of our self assembly process compared to methods relying on capillary methods, Section 3 analyses the relative importance of DEP and other forces intervening.

\section{Dielectrophoretic force}

We concentrate in this Section in the study of the dielectrophoretic force (DEP) in the self-assembly process of electrosprayed nanoparticles. This is an electrokinetic force that originates in the electric field present in the electrospray deposition process.

Figure 2 shows a schematic view of the electrospray geometry where a electrically conductive needle is fed with a nanofluid of nanoparticles in colloidal solution.

The tip of the needle is located at a distance D of a substrate. After a high DC voltage, in the range of several kilovolt, is applied between the needle and the substrate, an electrospray process is developed consisting in 


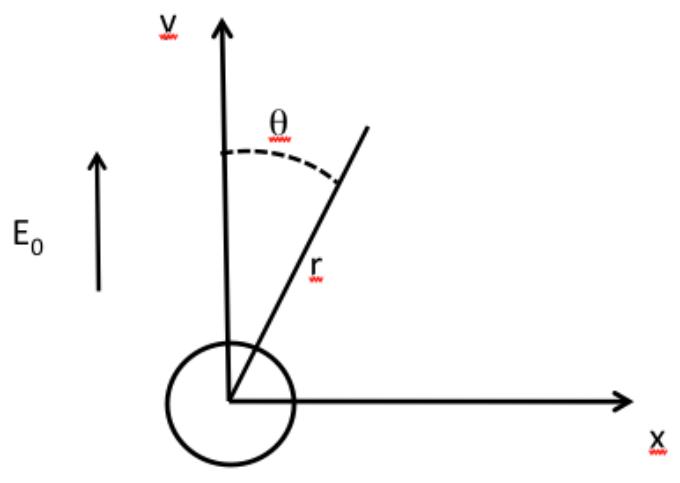

Figure 3: Geometry used in this work for a nanosphere located at the center and a second nanosphere located at the position $(\mathrm{r}, \theta)$

various phases. The first phase is the well known formation of a Taylor cone at the tip of the needle and its properties have been extensively studied [17], [18] . This is followed by the formation of a jet of electrically charged microdroplets, in our case containing nanoparticles in suspension, that are ejected following the electric field lines and the last phase is the production of ions in gas phase [19].

After travelling the distance D, the microdroplets land on the substrate and the phase of main interest to this work starts: the self assembly phase.

The several forces acting on the nanoparticles during this phase are weight, buoyancy ( when the substrate is wet), drag force, and at closer distances between particles van der Waals and double layer. The electric field originates electrophoretic and dielectrophoretic forces.The relative im portance of them are evaluated in Section 3.

Let us describe an anlaytical model of the dielectrophoretic force exerted on the nanoparticles as fundamental to explain the self-assembly phase.

Let us start by examining the simplest case shown schematically in Figure 3 in a two dimensional space, where a single lossless dielectric spherical nanoparticle of radius $\mathrm{R}$ is located at the centre of coordinates and is subject to a constant bias electric field $E_{0}$, directed as shown in Figure 3. Our calculation of the total electric field value in the surroundings of the nanoparticles follows the process described in [20] where the main simplifying assumption is 
that the particle can be represented by an electrical dipole having an effective dipole moment $p_{e} f f$. We are using this simplified model, as it is analytical, which is an approach also followed in reference [21]. We neglect multipolar terms described in [22], [23] and follow the approach as in references [24] and [25].

The electric field at any point in the plane identified by its distance to the center, $\mathrm{r}$, and by the angle $\theta$, is given by [24],

$$
\vec{E}=\vec{r}\left[E_{0} \cos \theta+\frac{p_{e f f} \cos \theta}{2 \pi \epsilon_{m} r^{3}}\right]+\vec{t}\left[-E_{0} \sin \theta+\frac{p_{e f f} \sin \theta}{4 \pi \epsilon_{m} r^{3}}\right]
$$

where $\vec{r}$ is the radial direction unit vector, $\vec{t}$ is the tangential direction unit vector and $\epsilon_{m}$ is the permittivity of the medium. The effective dipole moment $p_{\text {eff }}$ is given by [21],

$$
p_{e f f}=4 \pi \epsilon_{m} K R^{3} E_{0}
$$

where

$$
K=\frac{\epsilon_{p}-\epsilon_{m}}{\epsilon_{p}+2 \epsilon_{m}}
$$

is the Claussius-Mossotti factor and $\epsilon_{p}$ is the particle permittivity.

The dielectrophoretic force that the electric field exerts on another particle located at the point $(\mathrm{r}, \theta)$, and similar in nature to the one sitting in the centre of coordinates, is given by [20],

$$
F_{D E P}=2 \pi \epsilon_{m} R^{3} K \nabla|E|^{2}
$$

From equations (1) and (4) the dielectrophoretic force can be calculated as follows,

$$
\begin{aligned}
F_{D E P}=2 \pi \epsilon_{m} R^{3} K E_{0}^{2} & \left\{\vec{r}\left(-\frac{6 K R^{3}}{r^{4}}\left(2 \cos ^{2} \theta\left(1+\frac{2 K R^{3}}{r^{3}}\right)+\sin ^{2} \theta\left(-1+\frac{K R^{3}}{r^{3}}\right)\right)\right)+\right. \\
+\vec{t} & \left.\frac{2 \cos \theta \sin \theta}{r}\left(-\left(1+\frac{K R^{3}}{r^{3}}\right)\left(1+\frac{2 K R^{3}}{r^{3}}\right)+\left(-1+\frac{K R^{3}}{r^{3}}\right)^{2}\right)\right\}
\end{aligned}
$$

where the radial and tangential components of the forces are shown.

Figure 4 shows the values of the modulus of the dielectrophoretic force as a function of the angle $\theta$ for several values of the distance $r$ between centers of the two spheres $(2.5 \mathrm{R}, 3 \mathrm{R}, 5 \mathrm{R}$ and $10 \mathrm{R})$ considering that the medium is water and, for comparison, for $r=5 \mathrm{R}$ when the medium is air. The radius 


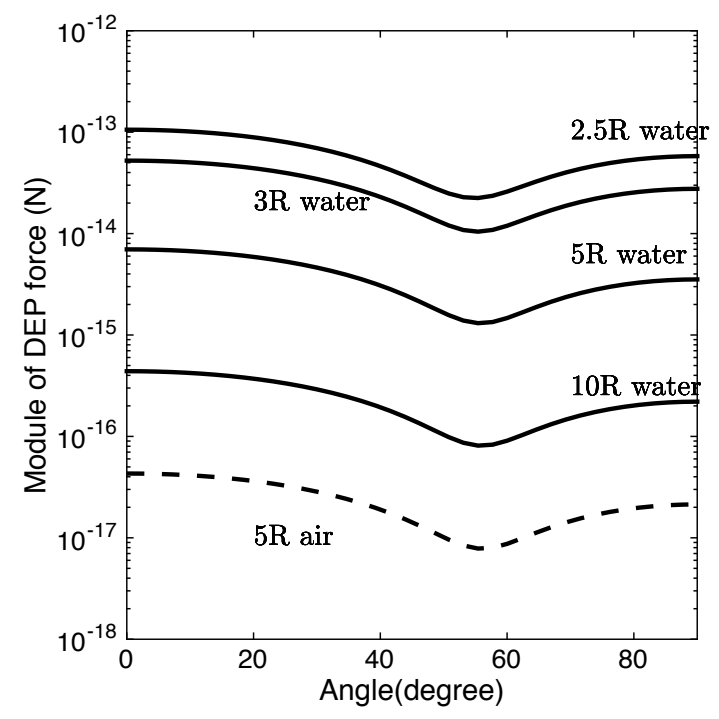

Figure 4: Magnitude of the DEP force at and angle $\theta$ at several distances between centers of two spheres in water as medium. For comparison the results ofr air at a distance of $5 \mathrm{R}$ is shown

of the spheres is $\mathrm{R}=190 \mathrm{~nm}$ and the material is latex $\left(\epsilon_{p}=2.5 \epsilon_{0}\right)$. As can be seen from Figure 4, the modulus of the force is strongly dependent on both the distance between the spheres - the force is stronger when the spheres are closer- and the kind of medium: in water more than two orders of magnitude stronger than in air. The effect of the medium is of course due to the different value of the Claussius-Mossotti factor and of the medium permittivity that together in the constant term in equation (5) gives a ratio water to air of 114.5 times, which is approximately the ratio of the two curves at $\mathrm{r}=5 \mathrm{R}$ in Figure 4. This two orders of magnitude difference can explain why our experimental observations are different from dry medium to wet medium. Moreover, in addition to the value of the modulus of the force, its direction is also relevant to the self assembly process. This is illustrated in Figure 5 where a polar plot of the DEP force is shown for two distances (3R and $5 \mathrm{R}$ ) and wet medium. As can be seen, there is a range of values of the angle $\theta$ where the net DEP force points towards the center ( where the other sphere is located) whereas in the rest is repulsive. The maximum value of 


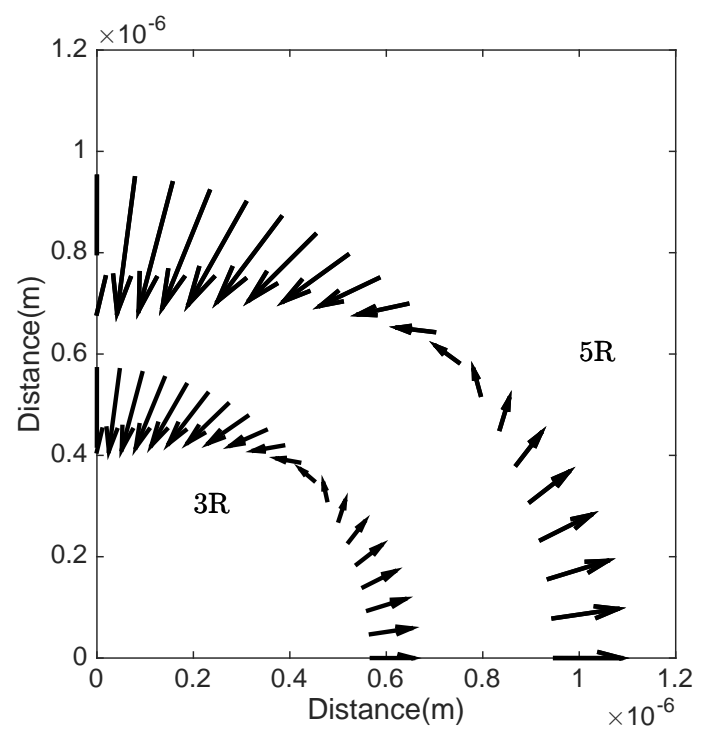

Figure 5: Polar plot of the DEP force on a latex particle located at the point $(\mathrm{r}, \theta)$ from the center where a similar particle is located. The distance between centers are $3 \mathrm{R}$ and $5 \mathrm{R}$

the force happens at $\theta=0$ and is pointing downwards, hence attractive, while for the angle $\theta=\pi / 2$ the net force is repulsive towards the right. This is consistent with the results shown in reference [26] [25] that include multipolar terms. This indicates that when the spheres are at close distances between $3 \mathrm{R}$ and $10 \mathrm{R}$ the most likely situation is the formation of chains in the vertical dimension that are kept a part from each other by the repulsive force in the horizontal direction.

A discussion about the validity of the assumption that the electric field $E_{0}$ can be assumed to be constant and having the direction shown in Figure 3 is relevant here. Although it may seem a crude approximation given the electrospray geometry and taking into account the high values of the voltage applied between the needle and the substrate, the fact is that the distances considered for the results shown in Figures 4 and 5 is restricted to some $10 \mathrm{R}$. For the nanoparticles considered here, of $\mathrm{R}=190 \mathrm{~nm}$, the region of interest is $1,9 \mu \mathrm{m}$. In order to have a quantitative assessment of the electric field values in the region we have solved the Laplace equation to find the 
electric field distribution in the geometry shown in Figure 1, considering an almost point electrode (the needle) and a flat substrate. To do so, we have used the Schwarz-Christoffel conformal mapping method [27] and closely followed the derivation for a focusing dielectrophoresis electrode described in reference [28]. Details are beyond the scope of this paper and will be reported separately.

Figure 6 shows the plot of the electric field components in the close vicinity of the substrate ( 2.5 micron). It can be seen that the vertical component has a value of $4.5 \times 10^{4} \mathrm{~V} / \mathrm{m}$ and quite constant in the range of hundreds of microns around the maximum that corresponds to the position of the needle $($ at $\mathrm{x}=0.05 \mathrm{~m})$. In the same location the horizontal component of the field has a zero at $\mathrm{x}=0.05 \mathrm{~m}$ and is positive to the right and negative to the left. Moreover the magnitude of the horizontal component is various orders of magnitude smaller, indicating that the electric field is almost vertical. In the simulations of the dipole equivalent model, we have considered that the bias electric field $E_{0}$ was $E_{0}=1 \times 10^{5} \mathrm{~V} / \mathrm{m}$, and thereby the results can be considered as representative of the real situation close to the substrate.

As the DEP force is proportional to the electric field module squared, as given in equation (4), the horizontal component is very small in the area of interest and the vertical component is negative and in the range of $6 \times 10^{10}$ $\left(V^{2} / m^{2}\right)$. From these results we conclude that our assumption above that the bias electric field $E_{0}$ in the vicinity of the substrate is constant and directed vertically is correct.

The analytical results shown in Figures 4 and 5 have been validated and extended using a multiphysics numerical software that solves the Laplace equation. Full 3D simulations are very costly in terms of computing time due to the wide range of distances involved that claim for a very large number of elements to get enough accuracy. However 2D simulations provide enough validation at this point of the study. Figure 7 shows the vector directions of the DEP force exerted on a particle having the centre in every point of the mesh up to when the spheres are tangent to each other.

We consider that there is a bias electric field of $E_{0}=1 \times 10^{5} \mathrm{~V} / \mathrm{m}$ and two latex spheres chained vertically. As can be seen, when the third sphere is located in the upper part of the plot, the DEP force tends to make the sphere to chain vertically with the other two. On the contrary, if the third sphere is located to the right of the plot, the dominant direction of the force is repulsive towards the right. This is consistent with the results in Figure 5 for just two spheres. It should also be noted that this repulsive force has 
smaller strength if the centre of the third sphere is horizontally aligned to the tangent point of the other two.

We next have examined the case of the two similar spheres tangent to each other but aligned horizontally at the centre of coordinates as shown in Figure 8.

Here we see that the third sphere, will be weakly attracted vertically and the centre will tend to go towards the tangent point of the other two spheres. From the results in Figure 7 we see that chaining horizontally is quite unlikely but in case that the distances between spheres are small is more likely that the third sphere will go towards the void in the middle of the other two.

In Figure 9 we examine the case of a chain of particles, vertically aligned. We consider that a third sphere of the same dimensions is placed close to the chain in different positions vertically and horizontally. The arrows indicate the direction of the force experienced by this third particle. We have used a vertical chain of 11 spheres to the left and another vertical chain of 5 spheres to the right of the plot, although only three spheres of the left chain are shown in the plot for clarity. Every point in the mesh shown is the center point of the 5 sphere chain of the right. We see that the left chain will exert a repulsive force against the right chain, thereby trying to keep them separated, but the strength of the repulsive force is weaker when there is a void-to-center alignment.

\section{Interpretation of the results}

The electrohydrodynamics of the electrospray process has been studied in depth, as mentioned earlier, since the discovery of the Taylor cone formation [17] at the tip of a needle when a high electric potential is applied between the tip and a substrate. Droplets are formed at the tip of Taylor's cone and disintegrate forming an aerosol of microdroplets. The microdroplets arrive to the bottom electrode, after a short lapse of time, where the self-assembly phase develops. After an initial splash transient, the nanoparticles undergo a sedimentation process in which several forces participate. If we consider that the liquid is slowly evaporating, the forces acting on the particles are: weight, buoyancy, friction, brownian, van der Waals, electrical double layer and electrokinetic (image, electrophoretic and dielectrophoretic). We next summarize this forces. 
Weight is a force normal to the substrate and is given by,

$$
F_{w}=\frac{4}{3} \pi R^{3} \rho_{p} g
$$

where $\mathrm{R}$ is the nanoparticle diameter, $\rho_{p}$ is the density of the particle and $\mathrm{g}$ the acceleration of gravity.

The buoyancy force is opposite in sign to the weight and is given by,

$$
F_{b}=\frac{4}{3} \pi R^{3} \rho_{m} g
$$

where $\rho_{m}$ is the density of the medium.

The drag force is given by ,

$$
F_{d}=6 \pi \mu R U
$$

where $\mu$ is the viscosity and $U$ the fluid velocity.

The van der Waals interparticle force is always attractive and between two spheres is given by [29],

$$
F_{v W}=-\frac{A}{6 R}\left(\frac{2(x+1)}{x^{2}+2 x}-\frac{x+1}{\left(x^{2}+2 x\right)^{2}}-\frac{2}{x+1}-\frac{1}{(x+1)^{3}}\right)
$$

where $\mathrm{A}$ is the Hamaker constant and $\mathrm{x}=\mathrm{d} / 2 \mathrm{R}$ being $\mathrm{d}$ the distance between the surfaces of the two spheres.

The electrical double layer force is always repulsive and is given by [30] ,

$$
F_{D L}=\pi \epsilon_{0} \epsilon_{m}\left(\frac{k_{B} T}{q}\right)^{2} Y^{2}(z) R^{2} \frac{1+\frac{z+2 R}{\lambda_{D}}}{(z+2 R)^{2}} e^{\frac{-z}{\lambda} D}
$$

where $\lambda_{D}$ is the Debye length, $\mathrm{z}$ is the minimum distance between the two surfaces , $k_{B}$ is the Boltzmann constant, $\mathrm{T}$ is the temperature, $\mathrm{q}$ is the electron charge and

$$
Y(z)=4 e^{\frac{z}{2 \lambda_{D}}} \tanh ^{-1}\left(e^{-\frac{z}{2 \lambda_{D}}} \tanh \frac{q \zeta}{4 k_{B} T}\right)
$$

where $\zeta$ is the zeta potential of the nanoparticles. The charge at the surface of the nanoparticles is given by

$$
\sigma=\frac{2 \epsilon_{0} \epsilon_{m}}{\lambda_{D}} \frac{k_{B} T}{q} \sinh \frac{q \zeta}{2 k_{B} T}
$$


This charge is subject to the electric field and hence subject to an electrophoretic force. Simultaneously, there is an electrophoretic retardation force originated in the hydrodynamic force exerted by the ions of the double layer in the solution drifted by the same electric field but in opposite direction. This retardation force reduces the velocity of the particles giving rise to an effective mobility that for thick double layers is given by,

$$
\mu_{e}=\frac{2 \epsilon_{0} \epsilon_{m} \zeta}{\mu}
$$

The process of droplet drying on a smooth substrate has been described in [31] where evidence of the competition between contact line pinning and dewetting creation patterns is shown. Moreover a single microsphere in colloidal solutions can pin the contact line if attached to the surface [32]. The electrophoretic force between polystyrene spheres with different surface chemistry was also studied [33] and it is proportional to the difference in zetapotentials. This force does not participate in our study as all particles have the same surface chemistry Movement of colloidal particles in a container with strip electrodes was investigated and anomalous movements at the edge of the electrodes were found [33]. The lift force experienced by an sphere in a viscous flow over an stationary plane is proportional to the $R^{4}[35]$ and to the flow squared, so in the dimensions we are considering here we can consider negligible the contribution of this levitation force. Figure 10 shows a plot of the value of the forces described above for a typical example of the values of the main geometrical parameters. We have considered the case of latex nanospheres with radius $\mathrm{R}=190 \mathrm{~nm}$, Hamaker constant $A=4.5 \times 10^{-21}$ [36], zeta potential $\zeta=40 \mathrm{mV}$, Debye length $\lambda_{D}=10 \mathrm{~nm}$. The values of the van der Waals, double layer and dielectrophoretic force in water and in air are shown as a function of the distance between the surfaces of two identical latex spheres. The DEP force is plotted at an angle $\theta=0$ for reference, although it is a function of the angle as described in Section 2. As can be seen, as the difference of the DEP force in water medium and in air is more than two orders of magnitude, in the range of distances considered in this plot, the DEP force can dominate.

\section{Conclusion and self-assembly model}

Let us first discuss the 'wet phase' of the deposition process. When the drops of the nanofluid have been desposited onto the substrate and the 
electrospray electric field is still $\mathrm{ON}$, the main forces playing a role in the close distances between spheres are shown in Figure 10 . As can be seen , the double layer repulsive force is counterbalanced by the attractive van der Waals force and in a range of distances the dielctrophoretic force dominates. It has to be taken into account that the weight is almost completely balanced by the buoyancy as the density of latex $(1040 \mathrm{Kg} / \mathrm{m} 3)$ and -water (999.97 $\mathrm{Kg} / \mathrm{m} 3$ ) are only different by a $4 \%$. Another potential force in this wet phase is the electrophoretic force itself due to the surface charge of the spheres . However this force is counterbalanced by the electrophoretic retardation force due to the opposite force exerted on the surrounding ions. We can estimate the drying velocity using the model that provides the instantaneous value of the radius of a droplet as a function of time by [37]

$$
R_{D}^{2}=k_{e} t
$$

where $R_{D}$ is the droplet radius $k_{e}$ is the evaporation rate constant and t is time. The dynamic change of the radius of the drop is then given by

$$
\frac{R_{D}}{d t}=\frac{k_{e}}{2 R_{D}}
$$

Using the value of $k_{e}=6.5 \times 10^{-9}$ to $6.5 \times 10^{-8} \mathrm{~m}^{2} / \mathrm{s}$ [37],for initial value of the droplet radius of $2 \mathrm{~mm}$, the value for derivative is $1.625 \times 10^{-6}$ to $1.625 \times 10^{-5} \mathrm{~m} / \mathrm{s}$. If we consider this value as representative of the drag velocity of the receding droplet surface this gives us drag forces between $5.15 \times 10^{-15} \mathrm{~N}$ to $5.15 \times 10^{-14} \mathrm{~N}$ that obviously dominate the forces when the spheres are separated by more than few microns. From the results shown we can conclude that the DEP force is quantitatively dominant in wet medium when the particles are close to each other. Additionally this force is highly directive tending to chain particles vertically and to assemble the chains void to center as they get closer.

\section{References}

[1] Sirelkhatim, A., Mahmud, S., Seeni, A. Aida N. Kaus M., Ann LC, Khadijah S. Bakhori M. Haan H. Dasmawati M. ' Review of zinc oxide nanoparticles : antibacterial activity and toxicity mechanisms'. NanoMicro Lett. (2015) 7: 219-242. 
[2] B.Véliz, S. Bermejo, A. Coll, and L. Castañer, Metal-insulator-metal capacitor using electrosprayed nanoparticles Applied Physics Letters 105, 013109 (2014); doi: 10.1063/1.4889746

[3] M. Maldovan ' Narrow low- frequency spectrum and heat management by thermo crystals' Physical Review Letters, 110, 025902 (2013).

[4] Y-H Ye, T.S. Mayer, I-C Khoo, I. B. Divliansky, N. Abrams, T. E., Mallouk, 'Self assembly of three dimensional photonic crystals with air core line defects, Journal of Materials. Chemistry, 12,pp.3637-3639 ( 2002)

[5] K. Ariga.Y. Yamauchi, T. Mori, J. P. Hill, 'What can be donde with the Langmuir-Blodgett method? Recent developments and its critical role in materials science, Advanced Materials, 25, pp. 6477-6512 (2013)

[6] J. Milette S. Relaix, C. Lavigne, V. Toader S. J. Cowling, I.M.Saez, R.Bruce Lennox, J.W. Goodby, L. Reven, ' Reversible long range patterning of gold nanoparticles by smectic liquid crystals' Soft matter, 8 , pp.6593-6598, 2012.

[7] J. D. Torrey, T. L. Kirschling L. F. Greenlee, ' Processing and characterization of nanoparticle coatings for quartz crystal microbalance, Journal of reseaerch of the national institute of standards and technology 120 , pp. 1-10 (2015)

[8] B.G. Jung, S-H Min, C-W Kwon, S-H Park, K-B Kim T-S Yoon ' Colloidal nanoparticle layer formation through dip-coating: effect of solvents and substrate withdrawing speed', Journal of the Electrochemical Society, 156, pp. K86-K90 ( 2009)

[9] A.Coll, S. Bermejo, D. Hernandez, L.Castañer' Colloidal crystals by electrospraying polystyrene nanofluids' Nanoscale research letters, 8:26 ( 2013).

[10] O.L.Salata ' Tools of nanotechnology: Electrospray' Current nanoscience 1, pp.25-33 ( 2005)

[11] J.B.Fenn, M. Mann, C.K. Meng, S.F. Wong, C.M. Whitehouse, ' Electrospray ioniuzation for mass spectrometry of large biomolecules Science, 246 (4926),pp.64-71 ( 1989). 
[12] P.J.Hull, J.L.Hutchison, O.V. Salata, P.J. Dobson, ' Synthesis of nanometer-scale silver crystallites via a room temperaute elecrostatic spraying process', Advanced Materials, 9, pp.413-417 ( 1997).

[13] C.H. Chen, M.H.J. Emond, E. M. Kelder, B. Meester, J.J. Schoonman ,' Electrostatic sol-spray deposition of nanostructured ceramic thin films', Journal of aerosol science, 30, pp. 959-967, (1999).

[14] S-H Hong, J.H. Moon, J-M Lim, S-H Kim, S-M. Yang, ' Fabrication of spherical colloidal crystals using electrospray', Langmuir, 21, pp.1041610421 ( 2005).

[15] [14] A.Coll, S. Bermejo, L.Castañer, ' Self assembly of ordered silica nanostructures by electrospray, Microelectronic engineering, 121, pp. 68-71 ( 2014).

[16] A Coll, S. Bermejo, I.Martin, R. ALcubilla, ' Laser contact trough electrosprayed alumina inverse opals on silicon', Microelectronic engineering, 145, pp. 29-31, (2015).

[17] M.S.Wilm, M. Mann, ' Electrospray and Taylor-cone theory, Dole's beam of macromolecules at last?', International Journal of Mass spectrometry and ion processes 136, pp.167-180 (1994).

[18] J. Fernandez de la Mora ' The fluid dynamics of Taylor cones' Annual Review of Fluid Mechanics, 39, pp217-243 (2007).

[19] T. C. Rohner, N. Lion, H. H. Girault, ' Electrochemical and theoretical aspects of electrospray ionozation ' Phus.Chem, Chem Phys, 6, pp.30563068 (2004)

[20] T. B. Jones ' Electromechanics of particles' Cambridge University Press ( 1995)

[21] N. Moghimi D. R. Decker S. Tatic-Lucic' Modelling and measurement of dielecrophoretic force and 2-D trajectories of microspheres in quadrupole electrode configuration' IEEE Sensors pp.1-4 (2012)

[22] T.B. Jones' Basic theory of dielectrophoresis and electrorotation' IEEE Engineering in medicine and biology magazine', pp.33-42 ( 2003) 
[23] T.B Jones' Multipole corrections to dielectrophoretic force, IEEE Transactions on Industry Applications, vol. IA-21, pp.930-934 (1985)

[24] C.Yang, Q.Ye, J.Li ' A modified dipole-enhanced approximation for a dielectric sphere', Journal of electrical and electronics engineering 5, pp. 1391-1394 (2005)

[25] Q. Ye, J. Li, J. Zhang' A dipole enhanced approximation for a dielectric mixture, Journal of electrostatics, 61 pp. 99-106 ( 2004)

[26] M. Washizu T.B. Jones 'Dielectrophoretic interaction of two spherical particles calculated by equivalent multipole-moment method' IEEE Transactions on Industry applications, 32, pp. 233-242 ( 1996)

[27] R. Schinzinger P. A. Laurra, 'Conformal mapping: methods and applications', Dover Eds., (2003).

[28] T.Sun, N.G. Green, H. Morgan ' Analytical solution for the electric field and dielectrophoretic force in a dielectrophoretic focusing electrode structure', Applied Physics Letters, 92, p.173901 (2008).

[29] H.C.Hamaker' The London-van der Waals attraction between spherical particles' Physica IV , 10, pp.1058-1072 (1937)

[30] J.E.Sader, S.L. Carnie, D.Y.C.Chan ' Accurate analytic formulas for the double layer interaction between spheres' Journal of Colloid and Interface Science, 171, pp. 46-54 (1995)

[31] [Deegan drying drop ] R. D. Deegan 'Pattern formation in drying drops' Physical review E, 61,pp. 465- (2000)

[32] G. D. Nadkarni, S. Garoff, 'An Investigation of Microscopic Aspects of Contact Angle Hysteresis: Pinning of the Contact Line on a Single Defect', Europhysics Letters,20, 523, 1992.

[33] D. Velegol, S.Catana, J.L.Anderson, S.Garoff' Tangential forces between nontouching colloidal particles' Physical review letters, 83, 6 pp. 1243-1246 (1999)

[34] J.Kim, S. Garoff, J.L.Anderson, L.J.M. Schlangen ' Movement of colloidal particles en two-dimensional electric fields' Langmuir , 21, pp. 10941-10947, ( 2005) 
[35] P. Cherukat, J.B. McLaughlin, ' Inertial lift on on a rigid sphere in a linear low field near a flat wall' Journal of Fluid Mechanics , 263, pp. 1-18 (1994)

[36] P Somasundaran, S. Shotri K.P Ananthapadmanabhan Mineral Processing: Recent Advances and Future Trends : Proceedings of a Conference Honouring Professor P. C. Kapur on his 60 birthday, Indian Institute of technology, Kanpur, December 11-15, 1995 pp. 126-137

[37] R. Kayser, H.S. Bennett, 'Evaporation of a liquid droplet'. Journal of Research of the National Bureau of Standards A- Physics and Chemistry, vol. 81A pp. 257-266 (1977) 

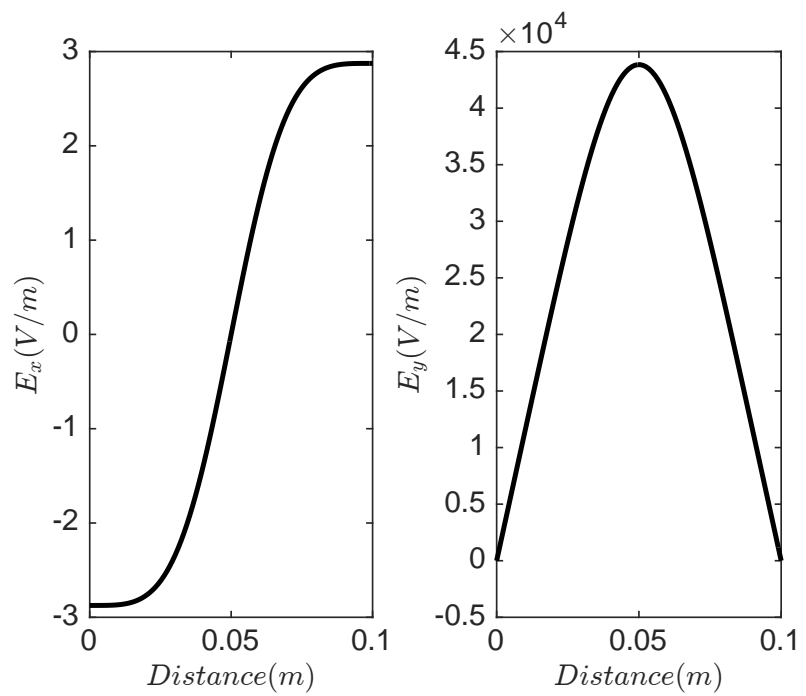

Figure 6: Main electrospray bias electric field components as a function of the lateral distance in the close vicinity of the substrate 


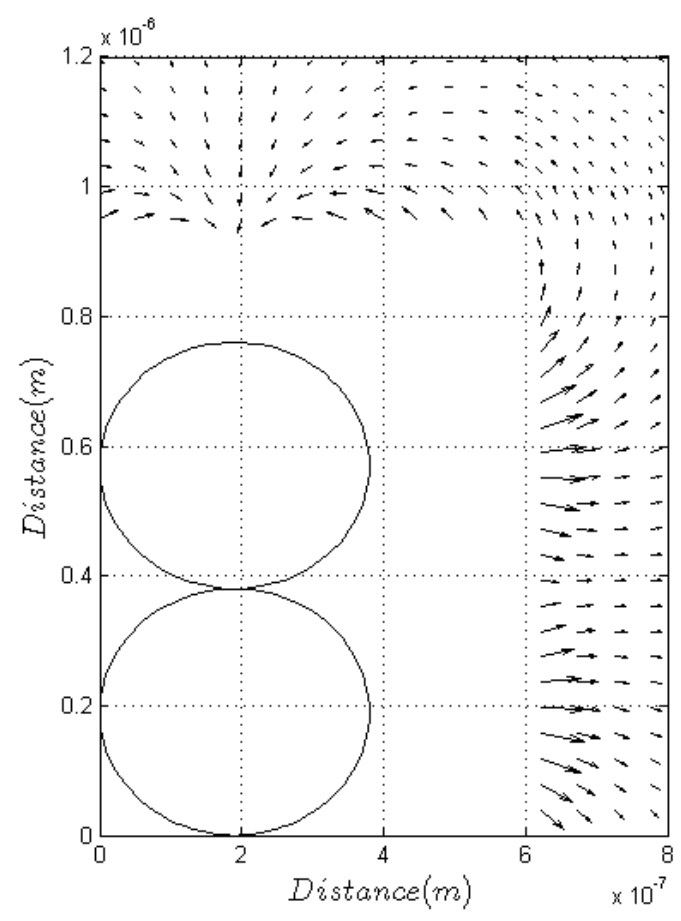

Figure 7: Plot of the vector direction of the DEP exerted on a latex sphere ( not shown) This third sphere has the center at points $(\mathrm{x}, \mathrm{y})$ located in the mesh shown at the starting points of the arrows. Two other identical spheres are located at the center and chained vertically 


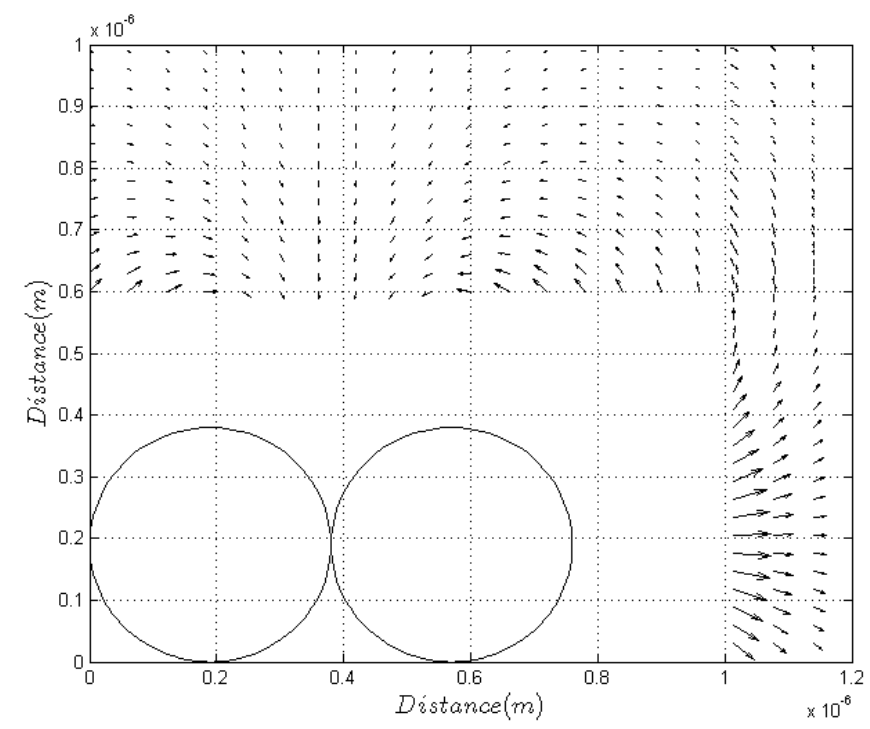

Figure 8: DEP force on a third sphere located at every point of the mesh when there are two other identical spheres chained horizontally 


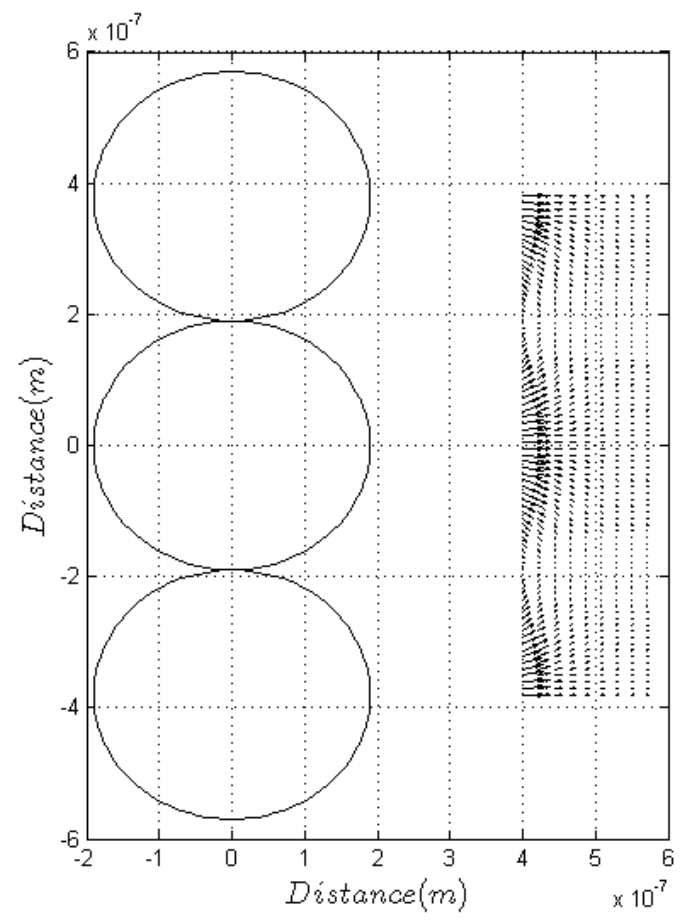

Figure 9: DEP vector force for the case of two vertical chains 


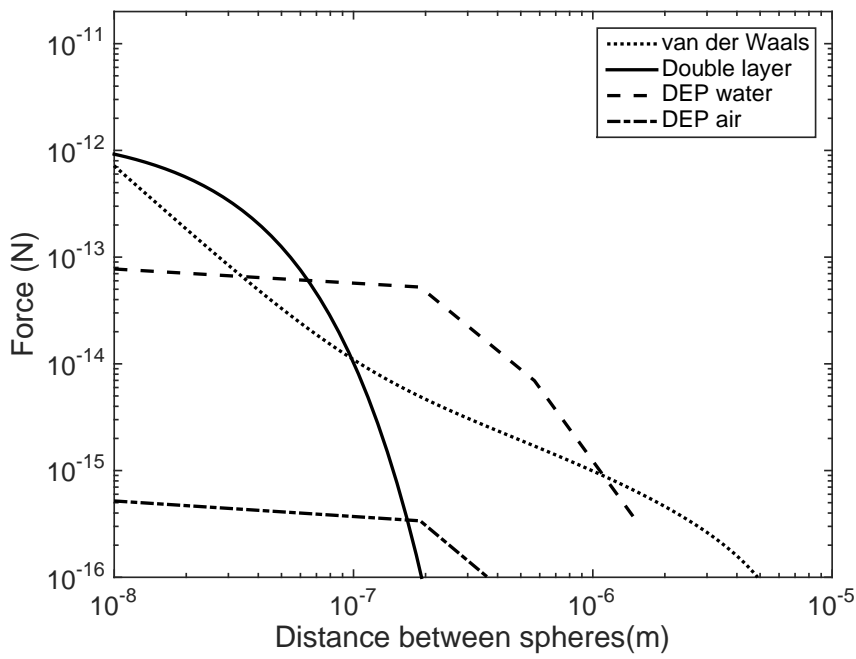

Figure 10: Plot of the main forces acting in the nanoparticles as a function of the distance between the surfaces of the spheres. DEP at $\theta=0$ are shown for wet and dry medium 